\title{
Research on Obstacle Avoidance System of UAV Based on Multi-sensor Fusion Technology
}

\author{
Deng Ke, Hou Xiaosong, Wan Wenjie, Liu Shiyi \\ State Grid Hubei Corporation Electric Power Maintenance Company, Wuhan, 430077, China \\ email: 1337967585@qq.com
}

Keywords: Multi-rotor UAV; Substation; Obstacle Avoidance System; Defect Recognition

\begin{abstract}
In view of the low efficiency and inspecting dead zone of inspecting in 500kV substation, a new inspection method is proposed, which is using UAV carrying multi-sensor detection devices instead of traditional manual inspection and robot inspection. The structure, the composition of obstacle avoidance system, route planning and the method of identifying the defect of substation equipment of multi-rotor UAV for $500 \mathrm{kV}$ substation equipment inspection are introduced in detail. In order to overcome the strong electromagnetic interference in substation, reasonable design of circuit layout, grounding design, filter design and shielding are designed, and carrier phase differential GPS satellite positioning system is chosen to realize the accurate positioning of UAV and surrounding equipment and improve UAV inspection and navigation accuracy in substation. The UAV patrol route is planned to ensure the safety inspection. the UAV carries visible light imaging device and infrared camera double patrol to distinguish substation equipment defects.
\end{abstract}

\section{Introduction}

At present, substation inspection mainly includes manual inspection and robot inspection, and manual inspection relies on patrol personnel to collect a large amount of operational data, which is not only affected by the quality of inspectors, technical level and environment, but also labor intensity, low efficiency[1-3]; robot inspection improves the status to a certain extent, but the current inspecting robots could only walk in accordance with the pre-laid path, and has high cost, low intelligence and inspection dead zone, due to the particularity of substation equipment $[4,5]$, some equipment could not be inspected effectively such as series compensation platform, the existing manual inspection and robot inspection can not solve this problem, there is blind spot inspection.

With the development of unmanned aerial vehicles (UAV) technology in recent years, UAV has gained a certain range of application in aerial power transmission line inspection, and have the advantages of close survey, different angle shooting, more complete data and higher work efficiency[6,7]. But UAV inspections is almost in the blank stage in the field of substation application. In the special compact space of the substation, because the substation equipment layout is compact, the insulation distance margins and other factors. The scheme of UAV inspection in the special compact space of the substation is designed, which could overcome the strong electromagnetic interference to achieve the normal flight. In addition, the obstacle avoidance system and the flight route is designed. The inspecting UAV inspects the $500 \mathrm{kV}$ substation equipment and identifies the substation equipment defect, which provide the key technical demonstration and support for the inspection and fault diagnosis and repair work of $500 \mathrm{kV}$ substation equipment.

\section{Overall Design}

The $500 \mathrm{kV}$ substation environment is complex, equipment space layout is compact, and the electromagnetic environment is complex with a variety of sources of interference, these factors led to that UAV has great difficulties in the field of substation inspection. In order to solve the above problems, a four-axis vehicle is chose which not only has the advantages of light weight, simple structure, fast maneuverability, convenient operation performance and high real-time inspection capability, but also has unique platform design to provide facilitate for processing units, sensors and 
other equipment.

Based on the characteristics of UAV in power inspection, the obstacle avoidance system based on multi-sensor technology (electromagnetic field detection sensor, ultrasonic ranging sensor and GPS receiver) is adopted to ensure the flight safety of UAV. In order to reduce the strong electromagnetic influence of the substation on the UAV, the system designs to select the appropriate device, a reasonable layout of the circuit and spectrum resource management, and use of grounding, harmonics and shielding measures to ensure the electromagnetic compatibility equipment. Carrier phase difference GPS satellite positioning system is selected to realize accurate positioning and improve the navigation precision of substation inspection UAV. Based on the voltage level of the substation, the electromagnetic environment, the height of the equipment, the surrounding buildings and the focusing distance of the UAV imaging equipment, the safe operation space is established for the UAV and the route planning of the substation is designed by BP neural network.

UAV inspection performed visible and infrared double inspection, real-time display video image of visible and infrared thermal image on a computer monitor or a master on a handheld device, focuses on key parts of the device for voice prompts, gives timely alarm for the device exception (Surface defects, temperature anomalies, etc.), and achieve automatic focus on image point of failure, while real-time storage the static image and dynamic video as the historical data archive. This complete fault identification mechanism can effectively identify substation equipment defects. The overall plan of 500kV substation equipment inspection UAV is shown in Figure 1.

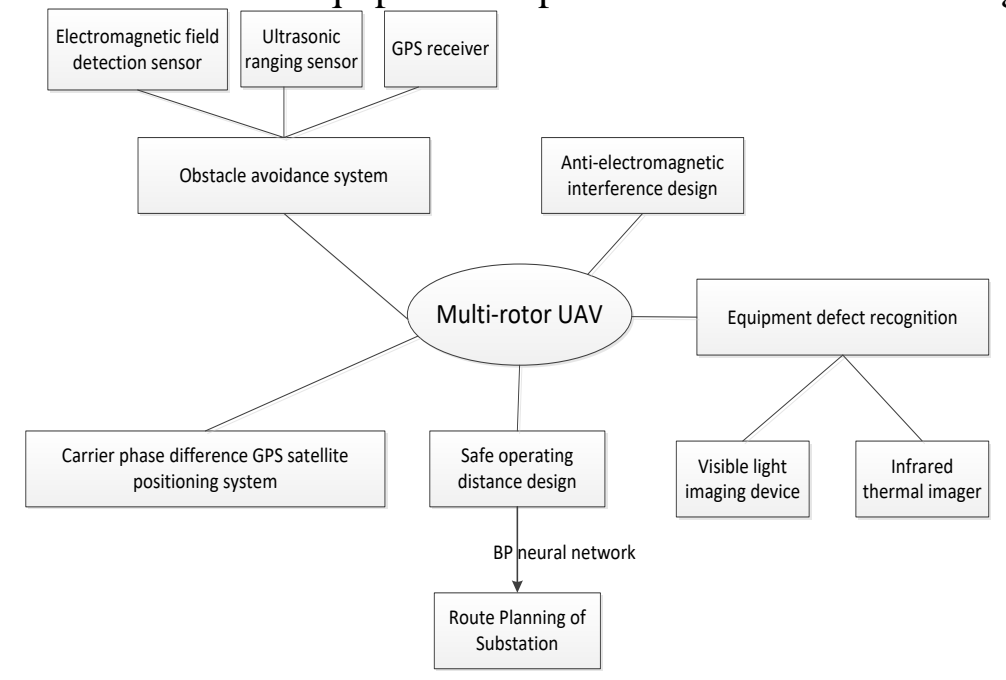

Fig.1 The overall plan of 500kV substation equipment inspection UAV

\section{Obstacle Avoidance System of Inspection UAV in Compact Space of Substation}

While the UAV inspects in the substation space, the surrounding environment is complex and varied, using a single sensor is difficult to accurately and comprehensively establish the surrounding environment model. Therefore, this paper uses multi-sensor information fusion technology to obtain information about the environment of UAV to ensure the flight safety. According to the characteristics of UAV in power inspection operation, the electromagnetic field detection sensor, ultrasonic ranging sensor and GPS receiver are selected to acquire the information needed by UAV to complete the UAV obstacle avoidance behavior. The multi-sensor UAV obstacle avoidance principle is shown in Figure 2. 


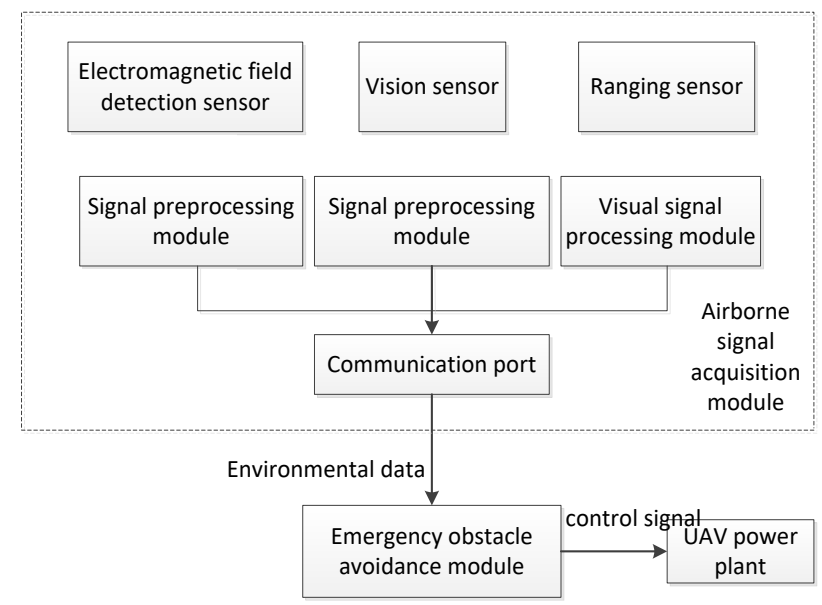

Fig.2 The multi-sensor UAV obstacle avoidance principle

The obstacle avoidance system of UAV is composed of position detection module, obstacle information processing module, embedded flight controller and airborne terminal of wireless data link.

(1) position detection module

Position detection module uses the combination of ultrasonic ranging sensor, electromagnetic field detection sensor and GPS receiver to determine the location and the relative position with the surrounding environment of UAV. The ultrasonic ranging sensor in the module is installed symmetrically around the UAV body. The GPS receiver obtains the longitude, latitude and altitude information of the current UAV. The electromagnetic field detection sensor obtains the electric field strength of the UAV current position. The ultrasonic ranging sensor acquires the distance between the UAV and substation equipment, power lines, and other obstructions.

(2) obstacle information processing module

The obstacle information processing module merges the data collected by the position detection module to provide the best obstacle avoidance decision for the UAV. Firstly, the obstacle information processing module preprocesses the data collected by the ultrasonic ranging sensor: comparing the data information of each sensor according to the performance parameters of the sensor, removing the wrong data and retaining the valid data, obtaining the distance between the UAV and the transformer substation equipment, the transmission line and the obstacle. Secondly, the obstacle information processing module preprocesses the longitude, latitude and altitude of the UAV collected by the GPS receiver, and then compares the data downloaded to the embedded flight controller, the distance between the UAV and substation equipment and the transmission line is calculated. Thirdly, the obstacle information processing module preprocesses the electric field strength information collected by the electromagnetic field detection sensor. Finally, the distance between UAV and substation equipment and transformer substation equipment is determined by comparing with the electric field distribution model which has been downloaded into the embedded flight controller and combining with the geographical position information obtained by the GPS receiver. The information of obstacle information processing module is processed by fuzzy neural network to get the obstacle avoidance decision-making and realize the obstacle avoidance of UAV.

(3) embedded flight controller

As the core of the UAV system, the embedded vehicle control system completes the flight control of the UAV. The main tasks include collecting the information such as attitude, position, angular velocity and acceleration of the unmanned aerial vehicle, and transmitting the collected information to ground monitoring Computer. And it needs to receive the instruction of the ground monitoring computer and carry out processing transformation on the command to realize the control of the UAV, save the electric field distribution model of the substation equipment needed for the obstacle avoidance control system. The embedded flight controller receives the obstacle avoidance decision made by the obstacle information processing module, and controls the obstacle avoidance behavior of the UAV. 
(4) ground monitoring computer

Its main function is to carry on the global path planning in the UAV flight area, and communicates with the ground segment through the airborne end of the wireless data chain to obtain the attitude information of the UAV, sends the control instruction to the UAV, realizes the remote control.

In the inspection process of UAV, it is possible to communicate with the airborne side through the ground end of the wireless data chain and transmit the electric field distribution model of the substation equipment and the database of the position information to the embedded flight controller. So as to facilitate the calculation of the obstacle information processing module. In addition, if the UAV nears the substation equipment, electromagnetic field detection sensor alarm information will be sent to the ground monitoring computer through the wireless data link. Ground monitoring personnel could also remote control of UAV based on UAV location information received by ground control computer.

\section{Accurate positioning and route planning in substation compact environment}

The inspection UAV selects the proper device, reasonable layout of the circuit and spectrum resource management, and adopts the measures of grounding, harmonics and shielding to ensure the compatibility of the equipment, for example, internal or inter-module wiring of each module of the equipment, printed circuit, secondary power supply, building wave module and shielded cable.

In order to realize the accurate positioning in the compact space of the substation, the carrier phase difference GPS satellite positioning system is selected in this paper. The positioning principle is shown in Fig.3. The system consists of a base station, a mobile station (GPS receiver) and differential data transmission system. Firstly, the coordinate information is measured by the reference station, and then the measured carrier observation and coordinate information are transmitted to the mobile station. The mobile station carries out differential calculation through observation information transmitted from the base station with its own observing information. Then the centimeter-level positioning accuracy could be got after real-time processing, which greatly improves the substation inspection UAV navigation accuracy.

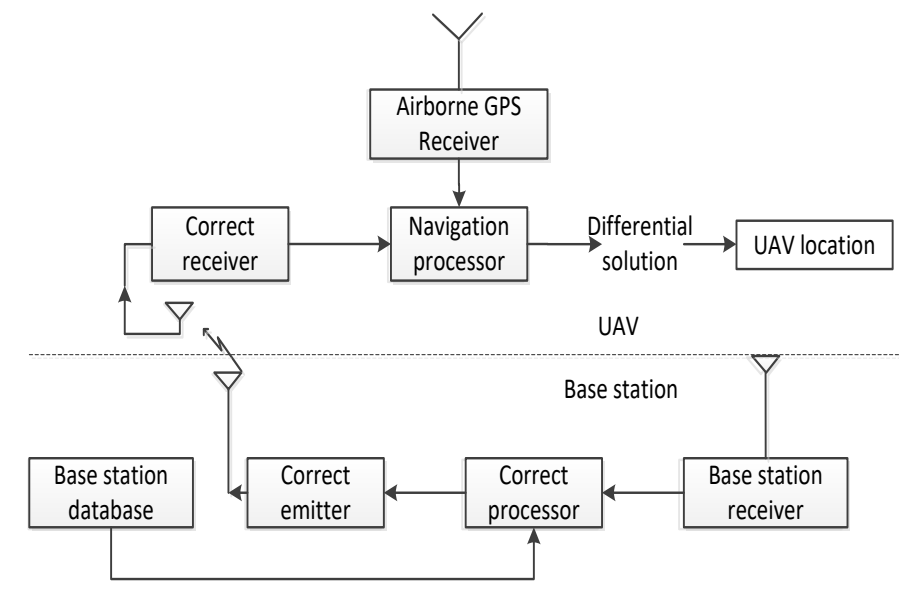

Fig.3 the carrier phase difference GPS satellite positioning system

The UAV automatic and manual remote control is achieved by setting waypoint, leg and home, as well as a specific flight strategy. The overall flight strategy is as follows: Set the minimum safety plane in the flyable space without obstructions, and UAV changes its observation points after vertically climbing to a safe plane height, flies straight to the next observation point above in the safety plane, then descends to the next observation point vertically, and so on to complete the normal timing of the surveillance. Directly selecting track points can temporarily switch for temporary observation of particular device. The flight strategy could apply home function, specify the home point to achieve alarm when the UAV is power shortage and automatically return to the home point. 


\section{Defect identification of substation equipment}

Substation equipment inspection multi-rotor UAV double inspects using the visible light imaging device and infrared thermal imager. The image visualization device carries out multi-angle, multi-azimuth grasping of the key equipment, image segmentation, edge detection and other image processing techniques for high-precision image stitching and fusion are selected to ensure that the image capture the clarity and accuracy. The visible light imaging device could identify surface defects such as corrosion, mechanical damage, connection of key parts, etc. there are always some trouble spots in the running process of faulty high voltage equipment,that is the thermal signs before the equipment failure, which could be remote, non-contact, real-time, fast accessed to by infrared thermal imaging. Using the substation equipment surface temperature and distribution of the test, analysis and judgment diagnoses equipment operation abnormalities and defects.

\section{Conclusion}

The multi-rotor UAV of substation equipment inspection could overcome the strong electromagnetic interference in the compact space of the substation, and inspect the defect of the substation equipment safely and efficiently. It provides a new idea for the inspection of the substation equipment and is of great significance.

\section{References}

[1] Zheng Weigang. Research on distribution network inspection system base on infrared image technology for UAV[D]. Shenyang Agricultural University, 2013.

[2] Xu Huadong. Research on Intelligent Obstacle Avoidance Methods for Power Lines Inspection with UAVs[D]. Nanjing University of Aeronautics and Astronautics, 2014.

[3] Wu Feilong, Lin Han, Tang Mingwen. Application of Multi-relay Method in Inspection of Large Unmanned Aerial Vehicle Transmission Line[J]. Electric Power, 2015,02:104-110.

[4] Liang Kun. Research on Ground Supervision and Control Technology of Power Line Inspection with Unmanned Aerial Vehicle[D]. Nanjing University of Aeronautics and Astronautics, 2014.

[5] Li Yong. Research on Application Mode of Unmanned Aircraft in Patrolling the UHV Transmission Lines[D]. North China Electric Power University, 2014.

[6] Gao Xu, Xie Jufang, Hu Dong. Application of Quadrotor UAV in the Inspection System of Citrus Orchard[J]. Process Automation Instrumentation, 2015,07:26-30.

[7] Wang Miao, Du Yi, Zhang Zhongyi. Study on power transmission lines inspection using unmanned aerial vehicle and image recognition of insulator defect[J]. Journal of Electronic Measurement and Instrumentation, 2015,12:1862-1869. 